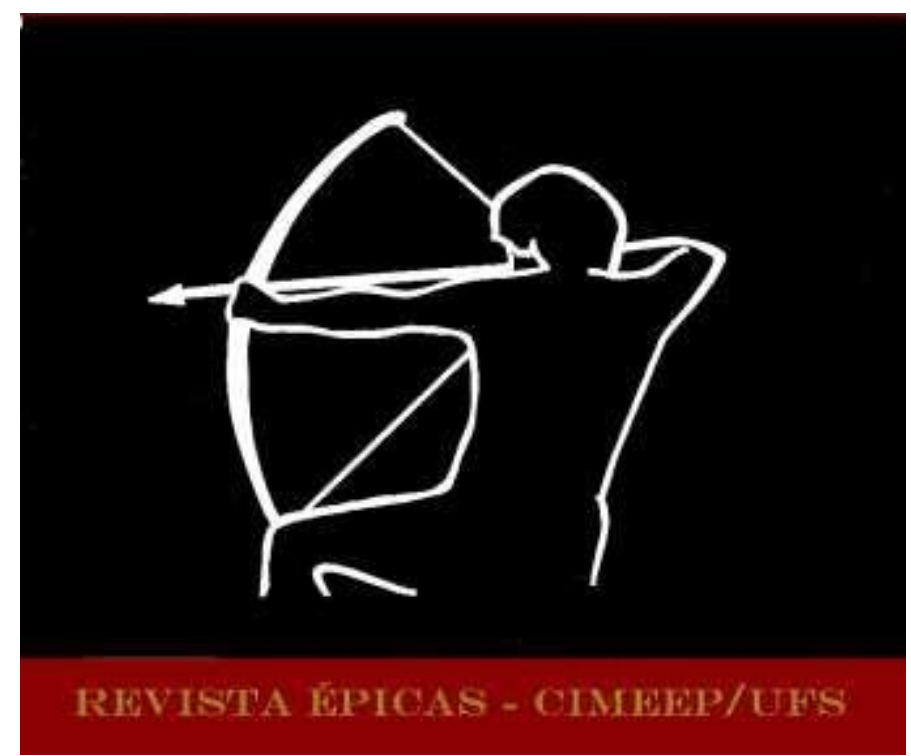

BRULEY, Julien. Essai sur une géographie évolutive dans l'épopée kirghize de Manas. In: Revista Épicas. Ano 5, N. 10, Dez 21, p. 30-44 . ISSN 2527-080-X.

DOI: http://dx.doi.org/10.47044/2527-080X.2021v10.3044

\title{
ESSAI SUR UNE GEOGRAPHIE EVOLUTIVE DANS L’EPOPEE KIRGHIZE DE MANAS
}

ESSAY ON AN EVOLUTIVE GEOGRAPHY IN THE KYRGYZ EPIC MANAS

Julien Bruley

CLERSE/SESAM, Université de Lille

Résumé: L'épopée de Manas est l'un des plus illustres représentants du genre épique en Asie centrale. Ses impressionnantes dimensions et sa vigueur en font un objet d'étude de choix pour de nombreux chercheurs. Le principal objectif de cet article est d'analyser certains aspects de la géographie épique manassienne et d'observer leur évolution au cours du temps. Le texte épique n'est en effet pas fixe ou statique, mais évolue en fonction d'un contexte particulier (historique, culturel, social), contexte qu'il adopte et adapte suivant les forces en présence. Le texte épique ne peut s'interpréter et se lire qu'en regard du contexte dans lequel il a été produit. Cet article s'interrogera sur le thème de la migration et de son éventuelle trace dans le texte, et montrera que l'épopée agit comme une matrice pour réfléchir un contexte ethnique et politique régional changeant. Un exemple concret, enfin, analysera les dissensions qui peuvent exister entre Chine et Kirghizstan au sujet du texte épique.

Mots clés: L'épopée de Manas, géographie épique manassienne, migration.

Abstract: The Manas epic is one of the most famous illustrative examples of the epic genre in Central Asia. Its impressive size and vigor make it an object of choice for many researchers. The main objective of this article is to analyze certain aspects of the Manas epic geography and to observe their evolution over time. The epic text is in fact not fixed or static, but evolves according to a particular context (historical, cultural, social), a context which it adopts and adapts according to the forces present. The epic text can only be interpreted and read by looking at the context in which it was produced. This article examines the theme of migration and its possible trace in the text, and shows that the epic acts as a matrix to reflect a changing regional ethnic and political context. Finally, a concrete example will analyze the dissensions that may exist between China and Kyrgyzstan on the subject of the epic text.

Keywords: Manas epic, Manas epic geography, migration. 
The "Manas" is an encyclopaedical collection of all the Kirghiz mythological tales and traditions, brought down to the present period and grouped round one person - the giant Manas. It is a species of Iliad of the Steppe. The Kirghiz mode of life, their morals, geography, religious and medicinal knowledge, as well as their relations with other tribes, all find illustration in this compendious epopee.

Čokan Valikhanov (1835-1865) ${ }^{1}$

Көбү жалган, көбү чын, Көрүп келген бирөө жок, Көрүнөө кудай билбесе, Жарымы төгүн, жарымы чын, Жараткан өзү билбесе, Жанынан келген адам жок. Sayakbay Karalaev (1894-1971) ${ }^{2}$

L'épopée de Manas est une œuvre majeure de l'orature kirghize (DOR, 2004), qui relate la lutte des Kirghiz contre de multiples oppresseurs, lutte menée par le héros éponyme Manas et sa truste de guerriers ${ }^{3}$. L'action épique se déroule essentiellement en Asie centrale et met en scène des populations ancrées sur ce territoire et se distinguant par leur ethnie, leur culture et leur religion. Considérée aujourd'hui par les Kirghiz comme l'épopée la plus longue du monde (une version enregistrée, éditée et publiée au XXe siècle dépasse les 500000 vers $^{4}$ ), elle reste toutefois assez peu connue, pour ne pas dire méconnue des spécialistes de l'épique. En effet, l'épopée comme texte n'est guère connue et étudiée en-dehors du Kirghizstan. Rarement mentionnée dans les publications internationales, elle reste cantonnée à des études localisées sur l'Asie centrale, et en particulier à des analyses portant sur le nationalisme kirghiz ou les liens entre l'épopée et l'idéologie d'Etat. Les études portant sur l'épopée elle-même - le texte - et ses récitants (les manasčl) sont rares, mais ont bénéficié d'avancées majeures depuis 1991. Enfin, parler de Manas n'est pas une action neutre. Le sujet reste remarquablement sensible au Kirghizstan dans la mesure où l'épopée, son héros, et les valeurs qui leur sont associées avec le temps, sont l'objet d'un éventail de définitions et d'interprétations parfois contradictoires, mais toujours partisanes : Manas, au Kirghizstan, n'est plus une simple épopée, mais un phénomène d'ordre socio-culturel (BRULEY, 2019). Cette dynamique, que j'appellerai contexte par la suite, joue un rôle prépondérant dans l'établissement du texte déclamé par les bardes. Dans cet article, j'aimerais introduire le lecteur francophone et un public plus large à cette épopée d'Asie centrale, et aussi présenter des réflexions dans le cadre de la thématique de ce numéro, en m'intéressant aux relations entre le texte épique et l'espace qu'il décrit et dans lequel il se déploie. Je ne m'intéresserai non pas seulement au texte mais également au contexte de celui-ci : ce contexte apporte en effet les clés de compréhension du texte, et peut expliquer certaines modifications, notamment d'ordre géographique, dans le texte. Dans une première partie, j’étudierai la question de la migration, ou du texte épique comme document historique.

\footnotetext{
${ }^{1}$ In MICHELL, 1865, 101. Čokan Valikhanov est le premier à avoir enregistré un épisode de Manas, en 1856, au cours d'une mission de reconnaissance en pays kirghiz.

2 Extrait de la version de Sayakbay Karalaev (MANAS, 2010, 354), un des plus célèbres manasči du XXe siècle, surnommé l'Homère kirghiz. Traduction : «Beaucoup de faux, beaucoup de vrai. Pas un n’a vu cela ! Si le dieu visible ne le sait, à moitié faux, à moitié vrai ! Si le Créateur lui-même ne le sait, aucun homme ne viendrait auprès de lui ! »

${ }^{3}$ Manas désigne par synecdoque une épopée comprenant trois cycles, Manas, Semetey et Seytek. Je me concentre ici principalement sur les textes manassiens du Kirghizstan. Il existe une tradition de Manas en Chine qui à elle seule mériterait un long développement, et soulèverait des problèmes d'un autre ordre. II en sera brièvement question en Partie III du présent article.

${ }^{4}$ Il s'agit de la version Karalaev, mentionnée en page précédente, et qui permet à Manas d'être considéré comme l'épopée - publiée

- la plus longue du monde (voir à ce propos les pages en russe et kirghiz sur le projet Wikipédia qui abondent en ce sens).
} 
J'établirai une comparaison fructueuse entre les premiers enregistrements de l'épopée au XIX ${ }^{\mathrm{e}}$ siècle et les textes obtenus après. Dans une deuxième partie, j'évoquerai la vision de soi et des autres dans les textes manassiens, et montrerai comment l'épopée absorbe la complexification du monde, de son contexte, et livre sa vision des choses. Enfin, nous nous pencherons sur un problème concret, celui des relations sino-kirghizes à travers le prisme épique. Comment une œuvre orale affecte des relations diplomatiques entre deux Etats voisins, la Chine d'une part et le Kirghizstan d'autre part ? Cette partie s'interrogera brièvement sur les interprétations de fiction et de véracité qui sont autant de partis pris dans ce problème.

\section{I) La géographie à l'origine de l'action épique}

Étudier le texte de l'épopée de Manas place le chercheur dans une situation périlleuse. Celui-ci pourra porter son attention sur un large choix de textes, plus ou moins anciens, mais enregistrés dans des conditions particulières, qui ne doivent pas être exclues de l'analyse, et qui constituent ce qu'on appelle le contexte. Dans le cas de Manas, ce contexte englobe la notion de performance, lieu et temps particuliers de la parole qui se donne, qui influence grandement la délivrance, la forme et le contenu du texte que le chercheur étudie. Dans cette partie, on passera en revue tout particulièrement certains changements structuraux du texte entre les versions enregistrées au XIX ${ }^{e}$ siècle, lorsque les Kirghiz cultivaient leur indépendance, et celles du XXe siècle où ils se trouvent intégrés dans la mécanique soviétique.

\section{Un monde réaliste}

Le prince kazakh et explorateur militaire Čokan Valikhanov est le premier à porter un regard sur le poème Manas ${ }^{5}$ et souligne l'ancrage de l'action épique dans l'actuel Kirghizstan du Nord, c'est-à-dire dans la plaine de la Chuy, dans la région du lac Issık-köl et de la rivière lli et jusqu'à Tachkent (VALIKHANOV, 1984, 327). II ne mentionne pas Talas qui est pourtant un des lieux les plus emblématiques de l'épopée. Cette connaissance localisée de l'épopée et son attachement à un territoire à la fois réduit (vallée de la Chuy) et élargi (mention de Tachkent, actuel Ouzbékistan) s'expliquent par sa rencontre avec une tribu kirghize dominante dans la région, en l'occurrence les Sarı-Bagıš.

Dans les fragments de Manas collectés par Vassili Radlov ${ }^{6}$ qu'il édita en 1885 dans le cinquième volume de ses Échantillons de littérature populaire des tribus turques $d u \mathrm{Nord}^{7}$, le cosmos épique se structure autour de deux axes, comme le précise Arthur Hatto : « dynamically a West-East axis stretching from Mekka, mythically conceived, to Barköl north of Hami, also mythically conceived. A North-South axis stretched from Kızîljar (Krasnoyarsk) to Kashgar" (HATTO, 1990b, 76).

\footnotetext{
${ }^{5}$ Et le premier à faire une comparaison avec les épopées classiques - et écrites - : Odyssée et lliade. Son expression « Manas est l'encyclopédie des Kirghiz » connaîtra une fortune particulière et est aujourd'hui la principale définition de Manas au Kirghizstan.

${ }^{6}$ Né Wilhelm Radloff, 1837-1918. On trouve les deux orthographes - l'allemande et la russe. Je conserve l'orthographe russe (Radlov) dans la suite du texte.

${ }^{7}$ En allemand, Proben der Volksliteratur der nördlichen türkischen Stämme. Une édition russe fut publiée en même temps à SaintPétersbourg. C'est elle qui contient le texte en kirghiz.
} 


\section{La naissance du héros : deux traditions}

Selon le lettré et historien kirghiz Belek Soltonoev (1878-1938), la naissance du héros est l'objet de deux traditions divergentes que nous qualifierons de géographiques. Soltonev rapporte en effet que " certains disent que Manas est né dans l'Altay, et d'autres disent qu'il est né à Talas » (SOLTONOEV, 1993, II, 136). Nous interpréterons ce propos de cette manière : il existe une tradition « fermée " ou "statique ", faisant naître le héros sur un territoire dominé par les Kirghiz ethniquement, politiquement et historiquement, et n'impliquant, par conséquent, qu'une importance limitée à l'espace comme origine ou justification de l'action ; et une tradition " ouverte " ou "dynamique ", qui place la naissance du héros en dehors de l'espace familier, au lointain, dans l'Altay, région associée par les Kirghiz, et donc leurs bardes, au territoire dominé par les Kalmak/Kalmuk (peuplade d'origine mongole désignant en langues turciques les Oirats). Le héros devra alors, dans un mouvement centrifuge, revenir vers sa terre natale, et le récit épique tendra à narrer cet effort.

Ces deux traditions semblent avoir connu des fortunes différentes avec le temps (période impériale, soviétique et indépendance), la dernière (dite "ouverte ») devenant la version la plus diffusée, voire canonique. Nous allons passer en revue quelques versions, enregistrées à différentes époques, afin d'observer leurs particularités et le rôle de cet exode dans l'action manassienne.

\section{a) La naissance de Manas (Radlov, enregistrée en 1869 ; publiée et traduite en 1885)}

Radlov, dans la préface au Tome 5 de ses Échantillons, expose en détail ses méthodes de collecte, sans toutefois fournir de renseignements sur l'identité des bardes auprès desquels il recueillit son matériau. Toutefois, il donne une anecdote très intéressante sur la manière dont il a enregistré la naissance du héros Manas :

The first episode, "Manas' Birth," which I recorded while I was staying with the Sary Bagysch south of Tokmak, was of meager content and seems to be a song triggered by my question about the birth of Manas. My question alone sufficed to urge the singer to a new song. (RADLOV, 1990, p. 81-2)

Cette version reste elle-même originale par rapport à la tradition « fermée » dans le sens où Talas (au Nord-Ouest du Kirghizstan actuel) n'est pas le siège du pouvoir de Manas. Au contraire, Manas naît dans les montagnes surplombant la ville de Vernoe (Almaty, Kazakhstan actuel). Ceci s'explique par le fait que Radlov a enregistré son épisode auprès d'un barde de la tribu des Sarı-Bagiš dont c'était le territoire. La naissance du héros s'inscrit donc dans un cadre quotidien et familier du barde, un espace réaliste et non mythique ou féérique. Enfin, on doit prendre en compte avant tout que la tradition « fermée » que Soltonoev centre sur Talas n'est mentionnée dans aucun des rares manuscrits qui nous soient parvenus de cette époque. L'épisode de Radlov constitue un exemple certes original, mais représentatif de cette tradition. 


\section{b) Dans les versions de Sagımbaj Orozbakov (1867-1930) et Sajakbaj Karalaev (1894-1971)}

Ces deux bardes sont considérés par les chercheurs kirghiz comme les plus importants du XXe siècle. Leurs versions sont présentées comme classiques, voire canoniques, et font l'objet de nombreuses études, les dimensions impressionnantes de leurs textes encourageant effectivement une pléthore d'analyses: Sagımbaj donna une version de Manas estimée à environ 180000 vers, et Sajakbaj récita les trois principaux cycles épiques en plus de 500000 vers. Bien que les deux bardes ne partagent pas le même parcours biographique - le premier est essentiellement un homme du XIXe siècle, le second est intégré au système soviétique (membre du parti communiste, chanteur de Manas professionnel à la Philharmonie) - leurs versions ont pu traverser l'époque soviétique et ses soubresauts, bien que leurs contenus diffèrent sur bien des points.

Les deux versions s'accordent et s'intègrent cependant parfaitement dans la tradition " ouverte ». Ce schéma respecte un motif particulier : les Kirghiz sont chassés de leurs terres - l'Ala Too ${ }^{8}$ - par leurs ennemis (dont le nom, Khitai, Mandchu ou Kalmak, diffère selon le barde, voir infra).

Dans ces vers de Karalaev, traduits par Elmira Köçümkulkızı (2005), on se rend compte de la situation catastrophique des Kirghiz, attaqués, submergés et forcés de quitter leur terre natale :

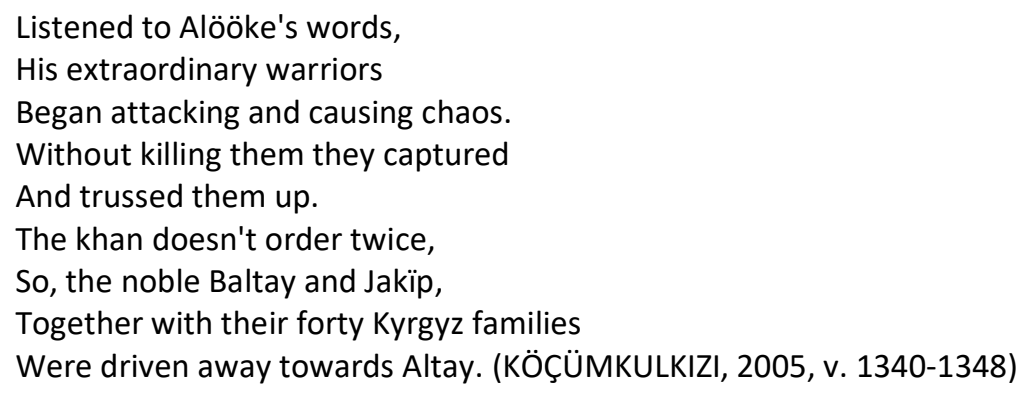

Dans ces deux versions canoniques, Manas, le héros, naît dans l'Altay et sa naissance présidera à sa destinée : il sera celui qui châtiera les ennemis des Kirghiz, et guidera son peuple vers sa terre natale. Une fois cette importante mission achevée, Manas attaquera à son tour ses ennemis sur leurs terres.

Cette version des choses, belliqueuse, est commune à la plupart des versions du XXe siècle et il semble qu'elle soit communément acceptée et partagée par les manasčı.

\section{c) Une exception, la version de Mambet Čokmorov (1896-1973)}

Ce manasčl est le seul, au XXe siècle, à localiser la naissance du héros au sein des frontières nationales (à l'époque, le Kirghizstan est une jeune république soviétique socialiste), mais dans un lieu original, que l'on peut, en un sens, rattacher à la tradition «fermée». La version de Čokmorov porte, selon ses commentateurs, de nombreux éléments féériques qui ne se retrouvent pas dans les versions canoniques et en font une œuvre unique. La naissance du héros, par exemple, ne s'inscrit pas dans un contexte de menace par des ennemis, mais dans le cadre d'une querelle familiale : Žakıp, le père de Manas, s'enfuit de son village 
afin d'échapper à une mort certaine. Après un itinéraire tortueux, il finit par s'arrêter à Arslanbap, célèbre forêt de noyers au sud-ouest du Kirghizstan, lieu qui verra naître Manas. Cette version constitue une exception. On la rattache, incidemment, à la tradition fermée, même si les motifs de l'action sont différents de ceux, habituels, de cette tradition (MANAS, 2017, p. 17).

\section{d) Une version contemporaine, Saparbek Kasmambetov (2011)}

Kasmambetov (1934-2020) est l'un des seuls manasčl à avoir été enregistré par des chercheurs occidentaux, et l'un des rares à avoir été traduit en anglais. Sa version - regroupant quelques épisodes cruciaux de Manas - fut enregistrée à l'initiative de Keith Howard, musicologue, traduite et publiée en 2011.

L'incipit est révélateur de son respect pour la tradition " ouverte ", où l'on retrouve la menace des ennemis, et le motif de la fuite pour la survie du peuple kirghiz :

I start with the birth of Manas, whose father was Jakıp, whose ancestor was Nogay, who had seven forefathers. Jakıp lived at the time when the Kalmaks from China invaded our lands. Our people fled their beloved pastures, heading west to the Altai Mountains. (HOWARD, KASMAMBETOV, 2011, p. 3) ${ }^{9}$

Et plus loin, on retrouve l'association entre la destinée du héros et celle de son peuple :

We have a Kyrgyz who can lead us. Now comes the time when we must gather our scattered people. Now we should prepare our arms, and make ready for when Manas comes of age. And so the Kyrgyz gathered and began to plan the journey back to their land, to the land of Talas. (ibid., p. 10)

\section{e) Une version de Manas contestée, Ajköl Manas (2014)}

Bübü Marijam kızı (Бүбү Мариям) est l'auteure, pour certains, d'une version de Manas, et pour d'autres d'un poème sur Manas, sans rapport avec l'épopée. Cette version et son auteure ont été vigoureusement attaquées par les universitaires. Quant aux manasčı, ils furent plus partagés sur la question ; une majorité reste toutefois hostile à cette version. En effet, cette version déroge à un certain nombre de règles (trame narrative différente des versions canoniques, versification originale, version écrite par une femme) qui ont entraîné, en 2016, une condamnation par voie de justice de cette œuvre.

Toutefois, quelle que soit la nature de cette œuvre, elle s'insère dans la tradition " ouverte " de l'épopée, à l'instar des versions classiques, en faisant naitre le héros dans l'Altai, à une date précise :

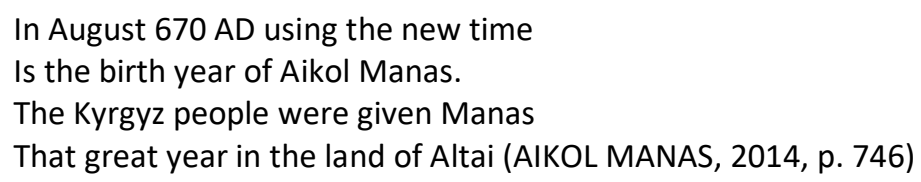

${ }^{9}$ La traduction est en prose. Le texte original versifié en kirghiz ne figure pas dans la publication, la publication se voulant « grand public » (communication personnelle de l'auteur, 2019). 


\section{Commentaires}

Il n'existe pas, à ma connaissance, d'étude sur la naissance du héros et d'explication concernant ce déplacement géographique dans la tradition épique. Plusieurs hypothèses peuvent être présentées : Hatto mise sur la loquacité du barde (en comparant la version Radlov et la version Orozbakov ${ }^{10}$ ) pour expliquer la longueur de cette action, et la capacité de ce même barde à insérer un effet dramatique que la version Radlov n'a pas. On peut remarquer également que la naissance du héros s'insère dans une destinée "négative " puis " conquérante » des Kirghiz chez Orozbakov et les tenants de la tradition " ouverte", tandis que la tradition "fermée " considère que la puissance kirghize (ou Nogoy, cf. infra) est déjà affirmée et va être renforcée par la venue du héros. On peut supposer que la tradition « ouverte » est d'apparition récente et prend sens si l'on place un contexte historique en regard du texte : les Kirghiz ont été petit à petit soumis au pouvoir impérial russe qui, en pacifiant la région, a imposé un ensemble de limitations de mouvements géographiques à des tribus nomades dont c'était le mode de vie. Cette limitation de l'espace explique en partie ce besoin d'extrapoler la naissance de Manas, mais ne fait pas pour autant des Russes le nouvel ennemi des Kirghiz. L'épopée procède à une recombinaison du contexte, non à un calque parfait de la réalité ${ }^{11}$.

\section{L'lenisseï dans le texte épique, un exemple d'influence scientifique}

Enfin, la présence de l'lenisseï dans le texte épique est un exemple daté et précis de l'influence des recherches scientifiques soviétiques de l'époque et de leur intégration dans Manas. Ce fleuve fait aujourd'hui pleinement partie de l'imaginaire kirghiz et se sa connaissance historique générale. Cependant, le fait que les Kirghiz proviennent de la région de l'lenisseï, puis qu'ils aient migré petit à petit vers l'Ala-Too, était inconnu des Kirghiz du XIXe et du début du XXe siècle, comme le précise Abdyldaev (ABDYLDAEV, 1995, II, p. 581). Deux versions seulement, au $X X^{\mathrm{e}}$ siècle, semblent avoir adopté cette information géographique et historique, celles de Ybyrajym Abdyrakman uulu (1888-1967) et de Žusup Mamaj (1918-2014), les deux personnages étant lettrés ${ }^{12}$. Cette tendance n'est pas largement répandue, mais est toutefois résiliente : le manasčı Talantaali Bakčiev (né en 1971), par exemple, est l'un des rares à en parsemer sa récitation. Cette intégration d'un fait géographico-historique nouveau dans Manas se limite essentiellement à une classe érudite parmi les bardes.

\section{II) Visions de 'soi', visions des 'autres'}

L'épopée de Manas offre un exemple très intéressant d'incorporation de la complexité du monde extérieur au texte épique et d'adaptation de ce contexte particulier à l'action épique. Le barde compose et recompose

\footnotetext{
${ }^{10}$ L'épisode 'la naissance de Manas' chez Radlov atteint les 164 vers et environ 12000 pour Orozbakov.

${ }^{11}$ Sur la présence des Russes dans les versions de Manas du XIX'e siècle, voir notamment Hatto et Prior. Des éléments sur cette même présence dans les textes du XXe siècle vont être présentés brièvement infra.

12 Ybyrajym Abdyrakman uulu est l'un des premiers Kirghiz à avoir été associé à la collecte de terrain du folklore kirghiz dès le début des années 1920. Les manuscrits de la version Orozbakov sont écrits de sa main. Žusup Mamaj est quant à lui le plus célèbre manasčı kirghiz de Chine. Sa version porte l'empreinte d'une connaissance vaste et érudite et n'est pas exempte d'une influence littéraire.
} 
en effet, au cours de la performance, un monde qu'il ne cesse de manipuler afin de donner à son audience un cosmos unique dans lequel évolue le héros. Mêlant réalisme et fiction, voire féérie, le texte déforme la réalité. Ainsi, le discours sur soi et le discours sur les autres interrogent le pouvoir de l'épopée à reconfigurer son monde et son idéologie sous l'influence de stimuli externes

\section{Manas devient Kirghiz}

L'un des changements les plus radicaux qu'ait expérimenté le texte manassien à partir des années 1920 est la cohésion que va gagner la structure du texte épique parmi les bardes. En effet, durant ces années les premiers folkloristes, sans véritable support étatique, et d'obédience djadide ${ }^{13}$, vont collecter et classifier le folklore kirghiz.

Si c'est grâce à ces actions volontaristes que l'on possède les versions de grandes dimensions du XX siècle, cette collecte n'est cependant pas détachée d'une certaine influence sur le contenu des textes collectés. En effet, parmi ces premiers folkloristes, on trouve le Bachkir Kayum Miftakov (1892-1948) qui enregistra, avec Ybyrajym Abdyrakman uulu, la version de Sagımbaj Orozbakov. Il est intéressant d'observer à ce propos l'apparition, dans le texte, d'éléments panturquistes, absolument étrangers à l'épopée, ainsi que la cristallisation d'une conscience "nationale » kirghize, qui va se traduire, dans le texte, par l'équation « Manas = Kirghiz ».

En effet, Manas était auparavant qualifié de Nogoy ${ }^{14}$. Les Nogoy sont, dans les épopées kirghizes et kazakhes, purement légendaires. Le nom se réfère historiquement à un arrière-petit-fils de Gengis Khan, Nogay Khan (mort vers 1300), régnant sur la Grande Horde. Le nom passa dans la tradition orale kirghize et finit par désigner des héros légendaires dont les faits guerriers sont estimés, tandis que l'ethnonyme Kirghiz, quand il apparaît, renvoie davantage à un présent plus trivial :

The traditional heroes of Kirgiz epic were Nogay, whose status as heroes par excellence resembled that of the Achreans in Homer [...]. It is touching that from the eminence of Kirgiz self-identification in song with the glorious Nogay, the old bards look down in scorn on the poverty stricken Kirgiz of real life on some of the rare occasions when they mention them (HATTO, 1977, p. 90).

À titre d'exemple, il est frappant de constater que l'usage de Nogoy est beaucoup plus fréquent que celui de Kirghiz dans la version de Valikhanov (17 pour 2$)^{15}$ et dans celle de Radlov ( 9 pour 7$)^{16}$.

Sagımbaj Orozbakov est considéré comme le barde qui a donné à Manas sa « nationalité » kirghize. Parler de « nationalité » peut paraître anachronique, mais les rapides et profondes mutations que l’État soviétique naissant va entraîner dans la région dans les décennies 1920 et 1930 autorisent un tel usage.

\footnotetext{
${ }^{13}$ Mouvement réformateur culturel et social musulman né dans l'Empire russe au XIX' siècle, qui fait porter son effort sur l'éducation et l'alphabétisme. Ce mouvement se développa en Asie centrale tsariste, mais disparut progressivement après 1917.

14 II existe différentes orthographes : Nogaj, Nogay, Noghay etc. J'emprunte ici la graphie kirghize.

${ }^{15}$ HATTO, 1977, 281.

${ }^{16}$ HATTO, 1990a, 622-3. Auxquels il faut ajouter 4 fois « Burut », terme qui désigne de manière dépréciative les Kirghiz (en langue mongole).
} 
L'épopée Manas sera justement appelée à plusieurs reprises " poème national » ou " épopée nationale ", chacune des nouvelles républiques d'Asie Centrale étant associée à une langue et une culture et une figure symbolique ou historique majeure (FOURNIAU, 2019, p. 215).

On peut expliquer la rapide adoption de cette équation « Manas = Kirghiz » où l'ethnonyme Kirghiz est utilisé positivement, par le fait que cette nouvelle interprétation du soi est le résultat de plusieurs processus (djadidisme, politiques des nationalités soviétique, etc.). De plus, l'apparition de nouveaux moyens de diffusion (tels les journaux) et d'une intelligentsia locale et lettrée, formée dans les premiers établissements scolaires soviétiques, a contribué à fortifier cette association et la rendre absolument indissoluble. Aujourd'hui encore, il n'est pas concevable pour un Kirghiz de donner à Manas une autre « nationalité » ni de le dissocier de son destin d'unificateur de la nation.

\section{Distinguer l'autre, distinguer l'ennemi}

Dans l'épopée, on observe également une reconfiguration de l'ennemi, de son identité et de ses caractéristiques. Les différentes versions respectent toutefois une distinction - réaliste, mais aussi caricaturale - à la fois ethnique, linguistique et religieuse. Les principaux antagonistes des Kirghiz sont les Kalmak (mongols Oirat) et les « Khitai » (Chinois) qui sont dans les textes souvent associés, voire confondus. Arthur Hatto décider de forger l'expression 'Sino-Kalmak' pour réunir sous un même terme les principaux des ennemis des Kirghiz (HATTO, 1977; 1990).

Les Sino-Kalmak se distinguent par un ensemble de traits caractéristiques des païens, opposés comme tels aux Kirghiz musulmans, mais aussi par la pression psychologique qu'ils exercent sur la nation kirghize : leur nombre surpasse largement les Kirghiz, et ils représentent une menace constante sur les marges du territoire kirghiz, principalement au nord - la région de l'Altay - et à l'est - la Chine moderne. À cette menace, Manas se doit de répondre avec force et d'assurer, par des attaques audacieuses, le contrôle de ces régions et de ces forces antagonistes. L'épisode La Grande Campagne (ou Čon kazat, expression qui emprunte le terme arabe gazat et donne à cet épisode militaire une dimension religieuse) est représentatif de cette réponse démesurée du héros à un danger du même acabit : Manas va se rendre maître de Beijin, la capitale lointaine des Sino-Kalmak ${ }^{17}$.

Au sud, la principale population qui peut jouer le rôle d'ennemi dans l'esprit de Manas est le groupe nommé « Sart ». Ce nom désigne, pour un nomade, le sédentaire, le citadin mercantile, le cultivateur, vivant principalement dans le khanat de Kokand et l'émirat de Boukhara. Ces deux territoires ont joué un rôle politique et militaire prépondérant en Asie centrale jusqu'à ce que l'empire russe les soumette. Dans l'épopée, ils sont parfois envahis ou menacés par Manas. Cependant, au-delà de cette vision manichéenne opposant nomade et sédentaire, l'épopée, dans toutes ses versions, apporte un élément original en mariant

\footnotetext{
17 Nous reviendrons en dernière partie sur cet épisode et ses conséquences pratiques.
} 
le héros Manas et Kanıkey, fille du khan de Boukhara, union qui scelle, symboliquement une alliance entre deux forces antagonistes.

Enfin, l'univers épique réaliste se double d'un versant féérique, lorsque l'action et le propos du barde s'éloignent du territoire bien connu de l'Asie centrale. La version Radlov, par exemple, évoque la défaite des Indiens (Inde) devant Manas - sans toutefois livrer de détails géographiques ou culturels à ce propos - et mentionne l'existence de confins habités par le Peuple de la Chèvre, le Peuple du Chien et le Peuple de l'Aurore, témoignant de l'insularité de la tradition épique du XIX' siècle (HATTO, 1990b, p. 82).

\section{L'épopée absorbe la complexité du monde}

Les textes épiques vont conserver cette dualité réalisme-féérique tout au long du XXe siècle, mais faire montre également d'une certaine avidité dans le savoir : le nombre d'ethnonymes ou de toponymes, dans les versions canoniques d'Orozbakov et Karalaev, s'élève à plusieurs centaines, sans que les chercheurs expliquent toutefois cette abondance et ses caractéristiques. En un sens, cette multiplication des peuples et des lieux peut s'expliquer par le contexte historique et sa complexification, que l'épopée tente, dans un mouvement de synchronisation, de comprendre et d'expliquer par le texte.

La version d'Orozbakov est à ce titre une riche mine d'informations puisqu'apparaissent des ethnies ou nationalités absentes des textes précédents; et ce phénomène ne peut se comprendre sans les mutations dont le barde lui-même a pu être témoin. En effet, le texte mentionne les Juifs (kg. žööt), les Allemands (kg. nemis < ru. nemets) et bien entendu les Russes (kg. orus). Cette absorption d'une multitude de peuples et de confessions différentes a, dans le cas d'Orozbakov, forcé le barde à réinterpréter la cosmogonie manassienne des textes du XIXe siècle. Le contexte n'est plus aussi simple qu'auparavant, et va jusqu'à entraîner, selon Daniel Prior, la possibilité d'un « relativisme culturel » (PRIOR, 2018, p. 242) dans le texte épique. Une autre conséquence remarquable, observée chez Orozbakov, est que le barde tend à rapprocher les Kirghiz des Kalmak sur un fond d'origine ethnique commune, les deux peuples sont, dans le texte, dits "fils de Türk ", ethnonyme (politisé) qui ne peut s'expliquer sans une influence extérieure sur le barde (ibid., p. 253).

Si le cadre épique offre un cadre immuable, une trame narrative généralement traditionnelle, celleci est donc régulièrement l'objet d'ajustements en fonction du contexte.

\section{III) L'épopée de Manas, pomme de discorde entre Chine et Kirghizstan}

L'épopée de Manas a, entre 1952 et aujourd'hui, été au cœur de certains problèmes d'interprétations de son texte, problèmes qui ont des conséquences pratiques dans son contexte. En effet, I'un des principaux ennemis des Kirghiz, dans le texte, sont les " Khitai », que l'on traduirait par " Chinois ${ }^{18}$ » en français. L'interprétation de ce terme est délicate, renvoyant à plusieurs strates historiques : il désigne tout d'abord un royaume fondé par les Khitai, dans le nord de la Chine actuelle, qui s'épanouit entre le $X^{e}$ et

\footnotetext{
18 Je garde le mot équivalent dans le texte français afin de ne pas surenchérir dans la mésinterprétation.
} 
le $\mathrm{XI}^{\mathrm{e}}$ siècles. Les populations turques en contact avec cet empire conservèrent le terme Khitay et le transmirent notamment aux Russes. Comme le précisé Louis Bazin,

En kirghiz actuel, « Chinois » se dit Kïtay, et les auditeurs Kirghiz de l'épopée de Manas ne peuvent spontanément comprendre ce mot que dans cette acception. D'autre part, l'association [...] du nom des Mandchous à celui des Kïtay ne peut guère se comprendre que par référence à l'Empire Chinois de la dynastie mandchoue des Ts'ing (1644-1912), dont la capitale, Pékin, était d'ailleurs celle des anciens souverains kitay (BAZIN, 1971, p. 379-80).

L'objet de ce chapitre est de montrer que le texte épique peut avoir des conséquences pratiques sur la géopolitique d'une part, mais aussi sur la politique culturelle internationale d'autre part. Tout texte épique se considère comme véridique et porteur d'une vérité historique. L'objet de cette partie est de passer en revue les limites que l'on peut donner à cette véracité, quand le symbolisme du texte doit être au contraire justifié et mis en avant.

\section{Le problème du texte dans un nouveau contexte}

En 1952, une campagne contre « le nationalisme culturel » fut menée dans les républiques à majorité musulmane : les épopées azéries, turkmènes, ouzbèkes, kazakhes et d'autres furent la cible du pouvoir central, victimes de ce que Benningsen (1975) a appelé la “ crise des épopées " ${ }^{19}$. Manas reçut le plus fort de l'attaque, mais fut aussi le témoin d'une résistance farouche de la part des intellectuels kirghiz. On notera que l'on attaquait l'épopée en tant que texte à valeur historique; on n'attaqua pas ceux qui la perpétuaient, à savoir les manasčı. En juin 1952, après une virulente campagne de presse entre " pro-Manas » et " antiManas ", une conférence fut organisée à Frunze, rassemblant près de 300 délégués du Parti communiste, de I'Académie des Sciences de I'URSS, de la Kirghizie, et des quatre autres républiques d'Asie centrale : deux camps se dessinèrent, mais aucune solution ne fut trouvée au niveau académique. Cette impasse fut toutefois contournée par le pouvoir central et Manas fut condamné ad vitam aeternam sous couvert de « panislamisme, nationalisme-bourgeois, aventurisme militaire et dédain pour les masses laborieuses ${ }^{20}$. La mort de Staline et la politique de Nikita Khroutchev permirent la lente réhabilitation des épopées d'URSS.

L'un des points de contentieux, parmi d'autres, en ce qui concerne Manas, touchait aux relations avec les Chinois (Khitai). En effet, c'était alors l'âge d'or des relations sino-soviétiques. La lutte contre les épopées centrasiatiques rencontrait, selon Vincent Fourniau, une question de politique internationale :

\footnotetext{
19 BENNIGSEN, 1975. Cette crise toucha non-seulement les épopées turques, mais aussi les épopées mongoles. Voir Roberte Hamayon, "The dynamics of the epic genre in Buryat culture ", in Epic Adventures: heroic narrative in the oral performance traditions of four continents, ed. JHM Jansen, Münster, LIT, 2004, p. 61. Bennigsen, qui est la principale source en ce qui concerne cette crise des épopées, a concentré son regard sur les populations musulmanes et turcophones de l'URSS.

${ }^{20}$ Bennigsen, 1975, 472.
} 
Les écrits actuels sur cette période font un lien entre l'établissement des relations de l'URSS avec la nouvelle Chine socialiste et le raidissement du pouvoir sur l'épopée, dans la mesure où le héros Manas guerroie sans relâche ses ennemis au-delà du Tian-Chian, en particulier les Djoungars, en territoire devenu chinois par la suite. Le lien entre appropriation patrimoniale et géopolitique fonctionne dans les deux sens dans l'esprit des dirigeants soviétiques. (FOURNIAU, 2019, p. 265)

Ce même contexte intervient au moment de l'indépendance du Kirghizstan en 1991. Le pays exerce une souveraineté nouvellement acquise, et Manas est le héros et symbole national, objet de festivités internationales célébrant son supposé millénaire en août 1995, financées en partie par l'Unesco (van der HEIDE, 2015, p. 25). Toutefois, le président se doit de procéder à certains réajustements afin de ne pas inquiéter son puissant voisin qui, I'URSS étant dissoute, se révèle comme un adjuvant et partenaire économique de choix pour un pays en reconstruction économique.

Le président Akaev dédie un chapitre entier de son livre L'Etat kirghiz et l'épopée nationale Manas (2002) à cette question. Sa démonstration, pour la résumer, vise à affirmer l'amitié entre la Chine et les Kirghiz dans le passé, amitié qui entérine, au présent, les bonnes relations entre la Chine et le Kirghizstan :

Parmi nos scientifiques, le problème du rôle de la Chine dans le destin historique du peuple kirghiz et de l'influence de la Chine sur la situation dans l'ensemble de la région n'a pas été abordé fermement. Les évaluations positives et négatives alternent, créant un " effet zébré ". Sans revendication scientifique aucune, j'exprimerai ma propre opinion sur cette question. Ma thèse principale est que les Kirghiz et les Chinois ne se sont jamais combattus dans leur histoire séculaire, qu'ils ne se sont pas non plus menacés, mais, qu'au contraire, ils ont cherché à établir des relations amicales réciproques à tous les stades de l'histoire (AKAEV, 2002, p. 352).

Puis, au cours d'un long chapitre, d'énumérer ces relations et contacts bienveillants entre les deux nations. Remontant I'histoire de la plus haute antiquité à nos jours, Akaev en vient à aborder des problèmes plus concrets et actuels, comme les conflits frontaliers. Toute sa démonstration ne visait qu'à cela, justifier sa politique dans la négociation des problèmes frontaliers avec la Chine (dans les années 1990) et, partant, de l'indéniable rôle de la Chine pour l'Asie centrale en général, et par le Kirghizstan en particulier en tant qu'allié économique.

Pourtant, après avoir affirmé avec force, lors des célébrations de 1995 et dans les années suivantes, le rôle de l'épopée dans l'histoire et les valeurs kirghizes, il évoque un passage particulier de l'épopée de Manas, I'un des épisodes les plus fameux, celui de 'La Grande Campagne' (Čon kazat - Чоң казат) :

Une question légitime se pose : pourquoi l'épopée évoque-t-elle les Chinois comme des ennemis de Manas, alors que les Kirghiz n'ont jamais été en guerre avec les Chinois, et n'y ont même jamais pensé ? La 'Grande campagne' à Beijing ne pourrait exister qu'en termes mythologiques. Dans un contexte purement psychologique, la situation est compréhensible: un grand héros épique comme Manas se doit d'avoir un antagoniste au moins égal à sa puissance héroïque. Et du point de vue du conteur, un tel adversaire ne pouvait être que la Chine. Le fait que le nom de la capitale uygur, Beitin, soit phonétiquement proche du nom de la capitale chinoise, Beijing, pourrait jouer un certain rôle. Jadis, quand l'art des bardes de l'épopée n'était pas restreint par le cadre politique et idéologique ${ }^{21}$, ils laissaient leur imagination débridée s'enfoncer jusqu'au cœur de la Chine et sa capitale Beijing (ibid., p. 414-5).

${ }^{21}$ Le lecteur remarquera l'évocation d'un cadre « politique et idéologique » qui pourrait restreindre l'imagination des bardes. On peut sans hésiter faire coïncider ce cadre avec la période soviétique. Mais une question se pose alors : maintenant que le président 
Maintenant que l'URSS n'est plus, les investissements chinois se font de plus en plus nécessaires, et nous voyons le président kirghiz louvoyer entre prétentions épiques et réalités géopolitiques et économiques : $c^{\prime}$ est que la Chine se dessine comme le nouveau puissant aux portes du petit Kirghizstan ${ }^{22}$.

Enfin, un récent article de Salamat Žıbıkeev ${ }^{23}$ fait le point sur les interprétations de l'épopée par les Kirghiz et les Chinois, et montre leurs apories. Comme Žıbıkeev le montre bien au début de son article, la question des relations entre les deux pays devient assez vite celle des relations entre deux peuples (people, народ) kirghiz et chinois. Cependant, Žıbıkeev n'évoque pas la présence d'une communauté kirghize en Chine, mais se concentre uniquement sur le texte de l'épopée, étudié conjointement par les chercheurs chinois et kirghiz : écrit en novembre 2019, l'article reflète la politique kirghize de ne pas intervenir dans des affaires qu'elle considère relever de la politique intérieure chinoise (en l'occurrence les " camps de rééducation » du Xinjiang).

\section{Une épopée, une ethnie, deux pays}

La Chine abrite la troisième plus grande communauté de Kirghiz après le Kirghizstan ${ }^{24}$, soit environ plus de 190000 personnes $^{25}$. Les Kirghiz sont présents dans le bassin du Tarim, depuis plusieurs siècles, et se sont retrouvés sous la coupe chinoise, officiellement en 1758. La Chine ne porta pas une attention marquée à cette région, si ce n'est pour y maintenir son autorité.

Au XXe siècle, dans les années 1950, la Chine ferma hermétiquement ses frontières, et principalement ses frontières terrestres occidentales. À partir de ce moment, la destinée de la langue kirghize, de part et d'autre de la frontière, ne fut pas la même. Les politiques linguistiques soviétiques et chinoises offrent quelques similarités (changements d'alphabets par exemple), mais les influences du chinois sur l'une, du russe sur l'autre, ont sans doute contribué à "fabriquer » des langues qui sont aujourd'hui sensiblement différentes. Le kirghiz de Chine s'écrit encore en arabe réformé, alphabet abandonné en Asie centrale soviétique à partir de 1928.

À cette divergence linguistique, il convient d'ajouter une distinction onomastique, touchant non l'ethnie, mais son nom. En effet, selon Rémy Dor et Guy Imart, en 1953, la République populaire de Chine

\footnotetext{
lui-même s'est expliqué à ce propos et a justifié des siècles de tradition orale en quelques phrases, cela signifie-t-il que Manas orientera sa Grande Campagne vers un autre ennemi dans les futures récitations des bardes?

22 II y aurait aussi toute une discussion à faire - qui n'a pas sa place ici - sur la vision des "Chinois " ou (kara) Kıtaj par les bardes Kirghiz des $X I X^{e}$ et $X X^{e}$ siècles qui reflètent, dans leurs récitations, une vision géopolitique qui leur est contemporaine. Voir notamment PRIOR, 2018, HATTO, 1977 et BAZIN, 1971.

${ }^{23}$ Salamat Žıbıkeev, "Epos "Manas" and Modern Kyrgyz-Chinese Relations », article en ligne : https://cabar.asia/en/epos-manasand-modern-kyrgyz-chinese-relations/, version russe : https://cabar.asia/ru/epos-manas-i-sovremennye-kyrgyzsko-kitajskieotnosheniya/ (article publié le 15/08/2019).

${ }^{24}$ Source: Petr Kokaisl, "Kyrgyz Minorities in China », Inner Asia, Vol. 14, No. 2 (2012), p. 384. En seconde position, on trouve l'Ouzbékistan.

${ }^{25}$ Estimation d'après le dernier recensement de la population chinoise de $2010: 186708$ Kirghiz (柯尔克孜族) étaient alors recensés. Source : https://en.wikipedia.org/wiki/List_of_ethnic_groups_in_China\#cite_note-4
} 
distingua ses Kirghiz dits 柯尔克孜 (« ke-er-ke-zi »; en kirghiz, кыргыз) des autres, étrangers - soviétiques dits 吉尔吉斯 («ji-er-ji-si »; en russe, киргизы $)^{26}$.

La trajectoire que l'on pourrait deviner pour les études chinoises de Manas dessine peut-être un déracinement de l'épopée de son territoire et de sa culture d'origine et sa promotion comme exemple de littérature proprement chinoise.

En 2009, la Chine inscrit à l'Unesco le Manas et la tradition épique de Gesar en son nom. Cette inscription provoqua un scandale au Kirghizstan dont l'une des principales conséquences est l'inscription, en 2013, par le Kirghizstan de l'élément « Manas, Semetey, Seitek : trilogie épique kirghize ». Cela constitue une réponse à ce qui fut considéré au Kirghizstan comme un vol de propriété culturelle ${ }^{27}$.

\section{Considérations finales}

L'épopée de Manas est un texte riche, dense et mouvant qui s'articule autour d'une trame dite classique, que les versions canoniques du XXe siècle ont plus ou moins entériné et fixé. L'évolution de certains aspects liés à la géographie épique démontre toutefois la dynamique du texte, qui essaye d'absorber un monde extérieur se complexifiant. L'un des problèmes majeurs auxquels est confrontée l'épopée au XXI siècle est celui de la fixité du texte. Si des modifications et l'intégration de nouvelles références spatiales ou de nouvelles ethnies ont pu donner au texte épique une sorte d'actualité, toute la question est de savoir si cette dynamique continue ou si, au contraire, le texte se sclérose et se sépare définitivement d'un monde bien éloigné de ce qu'il était pour les bardes du XIXe siècle. Ainsi, la géographie épique met l'accent sur la capacité du texte à se renouveler et à s'adapter à un contexte, mais également sur les limites de ces transformations.

\section{Références bibliographiques}

ABDYLDAEV, Èsenaly. Ene say. In: Manas Enciklopedija, tome 2, Bichkek, Muras, 1995, p. 581.

Aikol Manas (Manas Magnanimous), by Jaisan, Son of Umot Received from the living spirit and put down on paper by Mariam, Daughter of Musa, Volume 1, English translation by Adilet-Sultan Meimanaliev, 2014.

AKAEV, Askar. Kyrgyzkaja Gosudarstvennost' i Narodnyj èpos 'Manas' ('L'État kirghiz et l'épopée nationale Manas"). Učkun: Biškek, 2002.

BAZIN Louis. Histoire et philologie turques. In: Annuaire 1970-1971, Paris: École pratique des hautes études. $4^{\mathrm{e}}$ section, Sciences historiques et philologiques, p. 375-381, 1971.

BENNIGSEN, Alexandre. The Crisis of the Turkic National Epics, 1951-1952: Local Nationalism or Internationalism?. In: Canadian Slavonic Papers / Revue Canadienne des Slavistes, Vol. 17, No. 2/3, p. 463474, 1975.

${ }^{26}$ Guy Imart et Rémy Dor, Le chardon déchiqueté (être kirghiz au 20e siècle), Université de Provence, Aix-en-Provence, 1982, p. 8687.

27 Pour plus de détails sur la période 2010-2013 au Kirghizstan, et le déploiement d'une 'Manas mania', voir BRULEY, 2019. D'autre part, il convient de préciser que l'épopée de Manas est inscrite trois fois à l'Unesco, deux fois au nom du Kirghizstan (2003 et 2013) et une fois au nom de la Chine (2009). 
BRULEY, Julien. L'épopée de Manas : étude historique, patrimoniale et ethnographique. Thèse d'anthropologie sociale, Université de Lille, 2019, 666 p.

DOR, Rémy. Parlons kirghiz. Paris: L'Harmattan, 2004.

FOURNIAU, Vincen. Transformations soviétiques et mémoires en Asie centrale de l'«indigénisation» à l'indépendance. Paris: Les Indes savantes, 2019.

HATTO, Arthur Thomas. The Memorial Feast for Kökötöy-Khan (Kökötöydün ašı). Oxford University Press, 1977.

HATTO, Arthur Thomas. The Manas of Wilhelm Radloff. Otto Harrasowitz, 1990.

HATTO, Arthur Thomas. The Kirghiz and the Surrounding Peoples in Mid-Nineteenth Century Kirghiz Epic, in: DOR, Rémy. L’Asie centrale et ses voisins: Influences réciproques. Paris: INALCO, 1990, p. 73-83.

HEIDE, Nienke van der. Spirited Performance: The Manas Epic and Society in Kyrgyzstan. Bremen: EHV Academicpress $\mathrm{GmbH}, 2015$.

HOWARD, Keith and KASMAMBETOV, Saparbek. Singing the Kyrgyz Manas, Saparbek Kasmambetov's Recitations of Epic Poetry. Folkestone: Global Oriental, 2010.

IMART, Guy \& DOR, Rémy. Le chardon déchiqueté (être kirghiz au $20^{\circ}$ siècle). Université de Provence : Aixen-Provence, 1982.

KÖÇÜMKULKIZI, Elmira. The Kyrgyz Epic Manas. Selections translated, introduced and annotated by Elmira Köçümkulkïzï. disponible sur : http://www.silkroadfoundation.org/folklore/manas/manasintro.html

KOKAISL, Petr. Kyrgyz Minorities in China. In: Inner Asia, Vol. 14, No. 2, 2012.

MANAS. Version de Sahakbaj Karalaev. Sous la direction de A. Žajnakova, A. Akmataliev. Bichkek, Turar, 2010.

MANAS. Version de Mambet Čokmorov. Premier tome. Bichkek, Turar, 2017.

MICHELL, John and Robert (Tr.). The Russians in central Asia: their occupation of the Kirgiz steppe and the line of the Syr-Daria : their political relations with Khiva, Bokhara, and Kokan: also descriptions of Chinese Turkestan and Dzungaria. by capt. Valikhanof, M. Veniukof, and other Russian travellers. Translated from Russian, London : Edward Stanford, 6 Charing Cross, 1865.

PRIOR, Daniel. Sino-Mongolica in the Qırg̈ız Epic Poem Kökötöy's Memorial Feast by Saġımbay Orozbaq uulu. In: Philology of the Grasslands, Essays in Mongolic, Turkic, and Tungusic, Studies Series: Languages of Asia, Volume 17, 2018, p. 230-257.

RADLOV, Vassili. Preface to Volume V: The Dialect of the Kara-Kirgiz (Translated by Gudrun Böttcher Sherman, with Adam Brooke Davis), Oral Tradition, 5/1, p. 73-90, 1990.

SOLTONOEV, Belek. Kırgız tarıkhı: Tarıkhıy očerkter, 2 vol., Bishkek, 1993.

VALIKHANOV, Čokan. Sobranie sočinenij v pjati tomah, t. 1, Alma-Ata: Nauka, 1984.

ŽIBIKEEV, Salamat. Epos “Manas" and Modern Kyrgyz-Chinese Relations. In : Cabar, Bichkek, 15 août 2019. Disponible sur: https://cabar.asia/en/epos-manas-and-modern-kyrgyz-chinese-relations. Accédé en ligne le 15 mai 2021. 